\title{
Regulating the Regulators: The Post-Translational Code of Class I HDAC1 and HDAC2
}

\author{
Chiara V. Segré and Susanna Chiocca \\ Department of Experimental Oncology, European Institute of Oncology, IFOM-IEO Campus, Via Adamello 16, \\ 20139 Milan, Italy \\ Correspondence should be addressed to Susanna Chiocca, susanna.chiocca@ifom-ieo-campus.it
}

Received 27 July 2010; Accepted 15 October 2010

Academic Editor: Minoru Yoshida

Copyright ( 2011 C. V. Segré and S. Chiocca. This is an open access article distributed under the Creative Commons Attribution License, which permits unrestricted use, distribution, and reproduction in any medium, provided the original work is properly cited.

Class I histone deacetylases (HDACs) are cellular enzymes expressed in many tissues and play crucial roles in differentiation, proliferation, and cancer. HDAC1 and HDAC2 in particular are highly homologous proteins that show redundant or specific roles in different cell types or in response to different stimuli and signaling pathways. The molecular details of this dual regulation are largely unknown. HDAC1 and HDAC2 are not only protein modifiers, but are in turn regulated by post-translational modifications (PTMs): phosphorylation, acetylation, ubiquitination, SUMOylation, nitrosylation, and carbonylation. Some of these PTMs occur and crosstalk specifically on HDAC1 or HDAC2, creating a rational "code" for a differential, context-related regulation. The global comprehension of this PTM code is central for dissecting the role of single HDAC1 and HDAC2 in physiology and pathology.

\section{Histone Deacetylases: A Numerous Family with Many Biological Roles}

Histone deacetylases (HDACs) are modification enzymes that catalyze the removal of acetyl molecules from $\varepsilon-\mathrm{NH}_{3}$ groups of lysines, balancing the action of another family of enzymes, histone acetyl-transferases (HATs), which instead add acetyl groups.

They were first identified for deacetylating histones during chromatin remodeling, but many other nonhistone substrates have been characterized so far: transcription factors (p53 and $\mathrm{Rb}$ proteins), metabolic enzymes (Pyruvate kinase and Acetyl-CoA syntase), structural proteins ( $\alpha$ tubulin), enzymes involved in DNA dynamics (PCNA), as well as exogenous viral proteins (SV40 $\mathrm{T}$ antigen or HIV integrase) [1]. It is now clear that acetylation is a cellular broad-spectrum regulatory mechanism and HDACs are thought to be general deacetylation enzymes: in fact, it was recently proposed to rename them more properly "lysine deacetylases" (K[Lys]DAC) [2]. Nevertheless, since the name HDACs has historically entered into the acetylation field, in this paper we will refer to them as histone deacetylases (HDACs).

Histone deacetylases constitute an ancient enzyme family, conserved in evolution from yeast to plants and animals [3]. HDAC-like proteins are found as well in Eubacteria and Archaebacteria [4].

In humans, 18 HDACs have been described so far, divided into two families according to their mechanism of catalysis: zinc and $\mathrm{NAD}^{+}$dependent.

The zinc-dependent HDACs belong to the classical Rpd3/Hda1 family, while the $\mathrm{NAD}^{+}$-dependent HDACs belong to the Sirtuin family.

Another complementary criterion of classification is based on sequence homology to the corresponding Saccharomyces cerevisiae proteins. According to this classification, HDACs are divided into four classes.

Class I HDACs (HDAC1, HDAC2, HDAC3, and HDAC8) are homologous to the yeast RPD3 protein. Class II includes HDACs homologous to the yeast HDA1 protein, and is further divided into two subclasses: class IIa (HDAC4, HDAC5, HDAC7, and HDAC9) and class IIb (HDAC6 and HDAC10) [5]. Class III corresponds to the $\mathrm{NAD}^{+}$-dependent Sirtuin 
family and is composed of seven members, Sirt1-7 [6]. HDAC11 is the only member identified so far belonging to class IV; it is a zinc-dependent deacetylase sharing common features with both class I and class II HDACs [7].

The multiplicity of histone deacetylases reflects a diversification of functions in different tissues and biological processes. Sirtuins are mainly involved in metabolism and aging [6]; HDAC11 was shown to regulate the balance between immune activation and immune tolerance in $\mathrm{CD} 4^{+}$ T-cell [8] and plays a role in oligodendrocyte differentiation [9].

Class II members have a variety of different roles in muscle, heart, and bone development and physiology, and they are expressed with a certain grade of tissue specificity [10]. Class II HDAC6 is the main cytosolic deacetylase controlling the cytoskeleton dynamics and chaperone functions through acetylation of $\alpha$-tubulin and HSP90, respectively $[11,12]$.

On the contrary, class I HDACs are ubiquitously expressed in all tissue types [10]. HDAC8 plays an important role in smooth muscle cells, where it associates with $\alpha$ actin and is essential for cell contractility [13]. Recently, a relation between HDAC8 transcriptional overexpression and advanced or metastasized stages of childhood neuroblastoma was described [14].

HDAC3 has a role in cell cycle progression and DNA damage response [15], as well as in spindle assembly checkpoint and sister chromatid cohesion [16]. Germline deletion of HDAC3 is lethal and embryos die before day 9.5, while conditional knockout of HDAC3 in MEF cells affects the transcription of a variety of genes involved in metabolism, cell cycle, apoptosis, development, and signal transduction [15]. Liver-specific knockout of HDAC3 leads to hypertrophy of hepatocytes and dysregulation of lipid metabolism [17].

HDAC1 and HDAC2, which are strictly related proteins, also have crucial roles in development and physiology, especially in the heart and nervous system [18]. They are also deeply involved in cellular proliferation, cell cycle, and apoptosis. HDAC1 has a pivotal role in the regulation of the cyclin-dependent kinase inhibitor p21 [19], and knockout mice for HDAC1 are embryonic lethal due to a strong impairment in cellular proliferation [20]. Depletion of HDAC1 results in perturbation of the cell cycle with loss of mitotic cells and increase in cell death in different human cancer cell lines [21]. In this paper, we will describe in detail the regulation of class I HDACs, and in particular of HDAC1 and HDAC2.

\section{Regulation of Class I HDACs}

The activity of class I HDACs in the cells is regulated through three main mechanisms: subcellular localization, association with other proteins into multisubunit complexes, and posttranslational modifications (PTMs).

Class I HDACs are thought to be for the most part nuclear, with the exception of HDAC3, which possesses a nuclear export signal and can be found also in the cytoplasm $[22,23]$. HDAC1, HDAC2, and HDAC3 are usually localized on chromatin, except during mitosis when all the three
HDACs are excluded from compact, mitotic chromosomes $[15,24]$. The molecular details of how this exclusion is driven are still unknown.

HDAC1 and HDAC2, together with the histone binding proteins $\mathrm{RbAP} 46$ and RbAP48, are part of the catalytic core of many multiprotein transcriptional complexes, and this association stimulates their enzymatic activity [25]. The Sin3a and the NuRD complexes are broad-action modulators of gene transcription $[26,27]$ and bind a spectrum of different cofactors such as Sds3 [25] and SAP proteins [28], and Mi2, MTA2, and MBD3 [29], respectively. On the other hand, the REST/CoREST complex has more specific functions: it recruits HDAC1 and HDAC2 to suppress the transcription of neural genes in non-neural tissues [30, 31].

Recently, two other HDAC1/HDAC2 containing complexes were characterized; the SHIP and the NODE complexes. SHIP1 is a DNA-remodeling protein involved in chromatin dynamics during spermatogenesis: mass spectrometry analysis identified HDAC1 (but not HDAC2) as one of the components of the SHIP1 complexes [32].

NODE complex is peculiar of embryonic stem (ES) cells: HDAC1/HDAC2 containing complexes associate with the ES-specific transcription factors Nanog and Oct4, switching off specific target genes, and thus determining stem cell fate [33]. HDAC1/HDAC2 complexes are functionally distinct from HDAC3-containing complexes, such as the SMRT/NCoR complex [34], thus suggesting a differentiation of biological roles between HDAC1/HDAC2 and HDAC3. To date, HDAC8 has not been reported to associate in macromolecular complexes.

HDAC1 and HDAC2 are not only protein-modifiers, but they are in their turn widely post-translationally modified, as it will be exhaustively described in the following paragraphs. Post-translational modifications (PTMs) represent a versatile and rapid mechanism to modulate protein functions and properties, such as enzymatic activity, subcellular localization, stability, and interaction with other binding partners in response to different extra- or intracellular stimuli. They can be either chemical moieties (acetylations, phosphorylations, methylations, nitrosylations, ADP-ribosylations, glycosylations, and carbonylations) [35] or proteins (ubiquitin, SUMO, NEDD8, FAT10, ISG15, and Atg8/Atg12) [36].

It is possible that the key to dissect single-HDACspecific functions lies in decoding their PTM code, also, considering that, as it will be shown below, PTMs actively influence HDACs subcellular localization, enzymatic activity and complex formation. Given the above considerations, and given the strong association between HDAC1 and HDAC2 deregulation with the onset of many human diseases (from cancer to neuronal disorders, diabetes, and airway diseases), in this paper we will attempt to give a global overview of the post-translational code of HDAC1 and HDAC2.

\section{HDAC1 and HDAC2: Equal but Not Too Much?}

HDAC1 and HDAC2 are two highly related proteins, which are the result of the duplication of an ancient gene [5]. They 
share $85 \%$ of global sequence identity, but this identity is not uniformly distributed; the dimerization and catalytic domains, which comprise the N-terminal two thirds of the proteins (amino acids 1-325), are 92\% identical, while the C-terminal domains share $72 \%$ of identity. Even if the C-terminal domain has no catalytic activity per se, its deletion greatly reduces HDAC1 enzymatic activity [26], indicating that it plays an important role in the regulation of its functions. HDAC1 and HDAC2 are not only highly related in their structure and share many binding partners in macromolecular complexes (see above), but they have also redundant roles in a variety of biological processes. Conditional deletion of HDAC1 or HDAC2 was tolerated in different organs, such as the heart and the brain, if at least one allele of HDAC1 or HDAC2 was maintained [37], while concomitant deletion of both HDAC1 and HDAC2 caused mitotic catastrophe and cell death [38]. Differentiation of neuronal precursors into neurons requires the presence of either HDAC1 or HDAC2, strongly suggesting a compensatory effect [39]. Consistent with this observation, depletion of either HDAC1 or HDAC2 by RNA interference in cancer cell lines causes a compensatory upregulation of HDAC2 or HDAC1, respectively [21]. The redundancy of HDAC1 and HDAC2 has also been observed in the hematopoietic system. Levels and activities of HDAC1 and HDAC2 are critical for erythrocytic and megakaryocytic differentiation, and inactivation of HDAC1 and HDAC2 together results in severe thrombocytopenia due to apoptosis of megakaryocytes [40].

Nevertheless, the global picture is far more complex, because in other physiological settings HDAC1 and HDAC2 have specific and not compensatory functions. For example, knockout mice for HDAC1 die early in embryogenesis at $\mathrm{E} 9.5$ [20], while knockout mice for HDAC2 survive until the perinatal period [37]. It was reported that in HeLa cervical cancer cells RNA interference for HDAC2 results in an increase in p21 CIP/Wafl expression [41], while in other cell types, like osteosarcoma U2OS cancer cells, p $21^{\text {CIP/Wafl }}$ is upregulated also after HDAC1 depletion [21]. Finally, HDAC1, but not HDAC2, controls ES differentiation. Specific depletion of HDAC1 results in decrease activity of Sin $3 \mathrm{~A}, \mathrm{NuRD}$, and CoREST complexes, with concomitant decrease in acetylation of K56 of histone H3. Moreover, embryoid bodies derived from HDAC1-deprived (but not HDAC2-deprived) ES showed altered patterns of cardiomyocyte and neuronal markers [42].

The molecular rationale of when and why HDAC1 and HDAC2 have overlapping versus specific functions in different contexts is not yet clear. It is likely dependent on the cell or tissue types expressing specific combinations of proteins that can associate specifically with HDAC1 and HDAC2 or expressing different signaling pathways, in which PTMs likely act as principal effectors. It will be challenging to investigate whether a fine-tuned, differential PTM code might orchestrate differential functions of HDAC1 and HDAC2, thus creating a "single-HDAC regulatory code" despite their high overall similarity.

\section{The Post-Translational Modification Code of HDAC1 and HDAC2}

HDAC1 and HDAC2 are subjected to both chemical and protein PTMs. Although some PTMs occur in the catalytic domain, the majority of them occur in the less conserved Cterminal domain part (see Figures $1(\mathrm{a})$ and $1(\mathrm{~b})$ ). Known PTMs and their biological effects on HDAC1 and HDAC2 will be analyzed and compared.

4.1. Phosphorylation. Among the various post-translational modifications that occur on HDAC1 and HDAC2, phosphorylation is the best studied.

Phosphorylation in eukaryotes consists of the addition of a phosphate group $\left(\mathrm{PO}_{4}\right)$ on serine, threonine, or tyrosine residues by specific enzymes defined kinases. Phosphatases instead remove phosphate groups from proteins, balancing the action of the kinases [44].

HDAC1 is a substrate in vitro for phosphorylation by casein kinase II (CKII) and PKA but not by PKC, Cdc2, or MAP kinases [45]. HDAC2 is also phosphorylated by CKII in vitro, but unlike HDAC1, it is not a substrate for PKA [46].

The main kinase responsible in vivo for HDAC1 and HDAC2 phosphorylation is casein kinase II (CKII), which is also found in the HDAC1/HDAC2 containing complexes such as $\operatorname{Sin} 3$ and NuRD complexes [47]. It phosphorylates HDAC1 on serines 421 and 423 , and mutations in these sites determine reduction of deacetylating and transcriptional repression activities and interaction with $\mathrm{RbAP} 48$, Sin3a, CoREST, and MTA-2 [48].

It was also reported that CKII-dependent phosphorylation on HDAC1 is constitutive throughout the cell cycle, but dispensable for its intrinsic activity in vitro [49]. This was also confirmed in another study where HDAC1 treated with calf intestinal phosphatase is still able to deacetylate a histone H4-derived peptide [45]. An additional phosphorylation site on tyrosine $221 \mathrm{HDAC} 1$ was discovered through mass spectrometry screenings [46]: it is the only example of tyrosine phosphorylation on HDAC1, but without any evident role yet.

CKII also widely phosphorylates HDAC2 in vitro and in vivo on serines 422 and 424 , homologues of HDAC1 CKII-sites, and on serine 394, which seems to be the main phosphorylation site for HDAC2 [50]. Interestingly, one phosphorylation site was found in the region 386-409 of HDAC1 [45] and recent high throughput mass spectrometry analysis has identified the correspondent serine 393 of HDAC1 as a new phosphorylation site, but the role is still to be defined [51].

It was also reported for HDAC2 a fourth phosphorylation site on serine 411, but it seems to be independent from CKII action and its role is still unknown. In analogy with HDAC1, HDAC2 catalytic activity as well as the binding with Sin3a and Mi2 is promoted by CKII-dependent phosphorylation, while it does not affect the binding of HDAC2 with HDAC1 [50]. This is consistent with a previous study, which identified the interaction domain of HDAC1 with HDAC2 in the N-terminal region of HDAC1 [43]. 


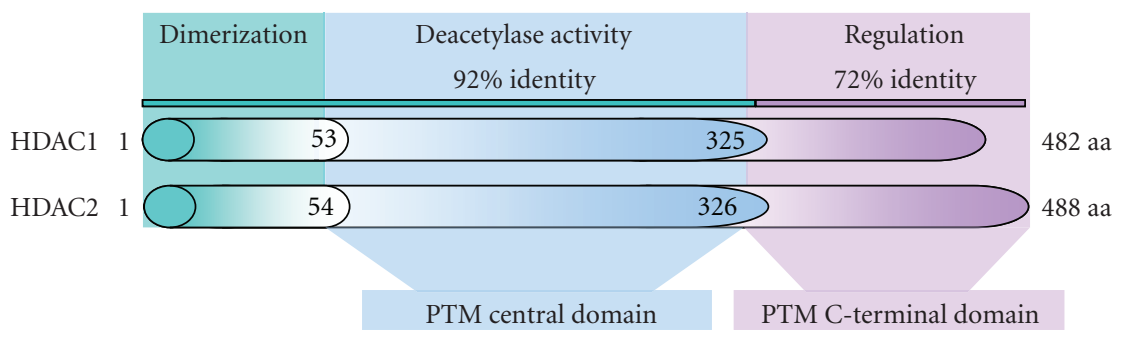

(a)

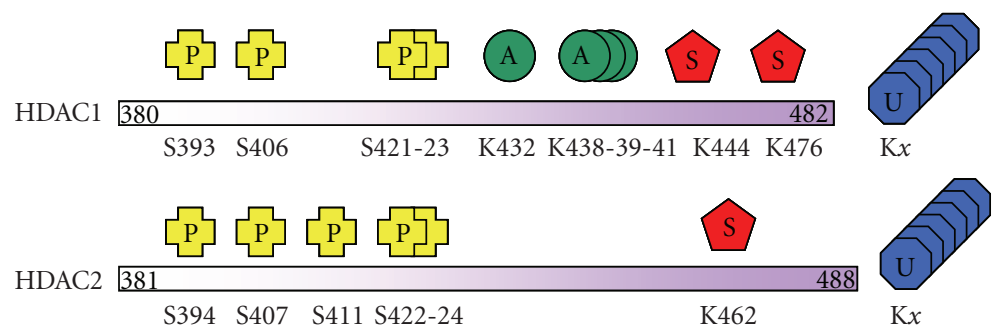

(b)

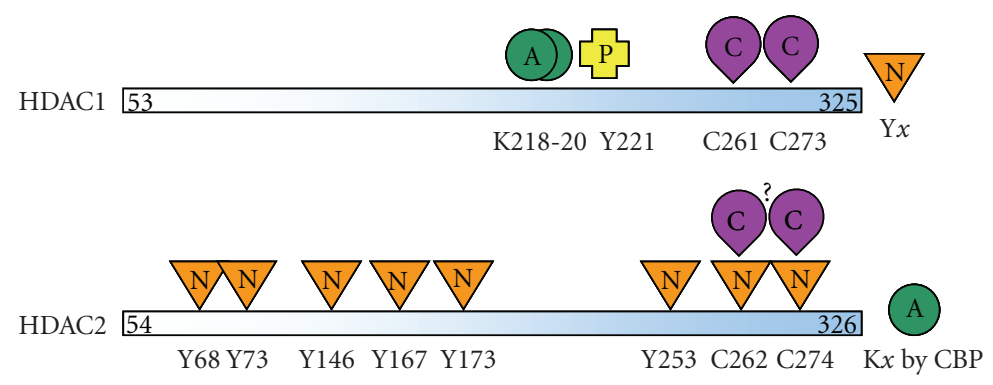

(c)

FIGURE 1: Schematic representation of the PTMs spatial distribution of HDAC1 and HDAC2. (a) Organization of the functional domains of HDAC1 and HDAC2, as described in [43]. Numbers indicate the corresponding amino acidic (aa) positions. Percentages of identity were calculated by BLAST alignment of HDAC1 (CAG46518.1) and HDAC2 (AAH31055.2) protein sequences. (b) and (c) Visual comparison of the different PTMs occurring on HDAC1 and HDAC2 in the C-terminal (B) and central (C) domains. Different PTMs are illustrated by different colors and letters: $\mathrm{P}$ (yellow): phosphorylation, A (green): acetylation, U (blue): ubiquitination, S (red): SUMOylation, $\mathrm{N}$ (orange): nitrosylation, and C (lilac): carbonylation. The amino acidic sites of modification are indicated by the number of their position in the protein sequence: S: serine, K: lysine, Y: tyrosine, C: cysteine, and CBP: CBP histone acetyltransferase. When the precise position of a modified residue is not known, the amino acidic site is followed by an $x$. The question mark (?) is used when the precise amino acidic site has not been formally identified. The amino acids at the boundaries of the two domains are reported.

CKII-driven phosphorylation of HDAC2 has been reported to regulate its differential distribution on specific functional areas of chromatin. In particular, unmodified HDAC2 is associated with coding region of genes contributing to active transcription, while the phosphorylated population is more involved in gene regulation, being preferentially recruited to gene promoters by RbAP48 into Sin3 and NuRD complexes $[47,52]$.

Phosphorylation of HDAC2 on serine 424 is also involved in the response of vascular smooth muscle cells to stimulation with all-trans retinoic acid (ATRA). Upon stimulation, a signaling cascade operating via the JNK kinase determines an increase in HDAC2 phosphorylation, which in turn causes the dissociation of HDAC2 from its binding partner KLF4. The consequent increase in KLF4 acetylation leads to the expression of differentiation marker genes such as SM22 $\alpha$ [53].

CKII-dependent phosphorylation of HDAC2 on serines $394,411,422$, and 424 increases upon exposure to cigarette smoke (CS) and is related to an increase in ubiquitination and degradation by the proteasome pathway in macrophage, human bronchial epithelial and small airways epithelial cells as well as in the lung of living mice [54]. Interestingly, single mutations of serine 394 or serine 424 did not affect global HDAC2 phosphorylation after CS exposure as the quadruple mutant did, suggesting a certain grade of redundancy between the sites [55]. Since HDAC2 suppresses expression of genes involved in inflammation, and cigarette smoke causes oxidative stress and is the primary risk factor for chronic obstructive pulmonary disease (COPD), the 
elucidation of this mechanism may have clinical relevance in the steroid resistance observed in COPD patients and in smokers affected by severe asthma.

The biological relevance of HDAC1 and HDAC2 phosphorylation is also depicted by the fact that some viruses induce their hyperphosphorylation in the early phase of infection. $\mathrm{U}_{\mathrm{S}} 3$ kinase from human Herpes Simplex Virus 1 [56] and its homologue ORF66 kinase from Varicella Zoster Virus [57] both induce, even if indirectly, hyperphosphorylation of HDAC1 and HDAC2. The target sites of ORF66-induced phosphorylation were identified as serine 406 of HDAC1 and serine 407 of HDAC2 [57]. Recently, also the nonhuman herpesvirus Pseudorabies Virus (PRV) $\mathrm{U}_{\mathrm{S}} 3$ kinase was shown to induce phosphorylation of HDAC2 on serine 407, indicating a conserved effect of many herpesviruses on this deacetylase [58]. The kinase(s) directly responsible for $\mathrm{HDAC} 1$ and $\mathrm{HDAC} 2$ phosphorylations in vivo is unknown; CKII seems unlikely to be the cellular kinase involved, since it requires the acidic consensus sequence S/T$\mathrm{X}-\mathrm{X}-\mathrm{E}$ [59], not present in the region encompassing serines 406 of HDAC1 and 407 of HDAC2.

CKII-dependent phosphorylation of HDAC1 and HDAC2 is also increased in response to hypoxic conditions, and this correlates with an increase in HDAC enzymatic activity [60]. These studies suggest that phosphorylation is a key signaling factor of different pathways that converge on HDAC1 and HDAC2 to carry on biological responses.

Finally, it was shown that proper histone deacetylation is required for meiotic resumption in porcine oocytes, and phosphorylation of $\mathrm{HDAC} 1$ was observed to rapidly change during oocyte maturation. The role of HDAC1 phosphorylation in the meiotic process has not been investigated in details but it is another indication of the variety of biological processes where phosphorylation of HDAC1 is involved [61].

Protein phosphatases are as important as protein kinases in regulating the phosphorylation status of substrates. HDAC1 and HDAC2 are dephosphorylated in vitro by protein phosphatase 1 (PP1) and $\lambda$-phosphatase [62]. Treatment of different cell lines with the phosphatase inhibitor okadaic acid induces the appearance of hyperphosphorylated forms of HDAC1 and HDAC2, associated with a slightly decrease in HDAC activity and loss of binding between HDAC1 and Sin3a and HDAC1/HDAC2 with the transcriptional partner YY1 [62]. Apparently, this seems in conflict with the work described above showing that phosphorylation positively regulates HDAC1 and HDAC2 enzymatic activities and binding properties. It is worth pointing out that the different studies used different approaches (site-directed mutagenesis versus treatment with a general phosphatase inhibitor), and, considering the complexity of the system of modifications acting on HDAC1 and HDAC2, this could at least in part account for the apparent discrepancy. HDAC1 and HDAC2 interact with protein phosphatases in vivo $[63,64]$; treatment of cells with the pan HDAC inhibitor trichostatin A (TSA) [65] causes destruction of a HDAC1-PP1 complex [66, 67], but the catalytically dead mutant of HDAC1 (HDAC1 H141A) still retained binding with PP1 [63]. This example shows that the use of aspecific, general inhibitors of the enzymatic activity of an enzyme (TSA or okadaic acid) may have different effects compared to the use of more specific point mutants.

HDAC1, and to a lesser extent HDAC2, were found in complex with PP1 and the transcription factor CREB. HDAC1 and PP1 cooperate to deacetylate histones and to dephosphorylate CREB, thereby silencing CREB-driven transcription in c-AMP unstimulated cells or in later times after stimulation, contributing to signal attenuation [63]. HDAC1 and PP1 are also part of a complex with the $\mathrm{Rb}$ protein, which is dissociated in response to ionizing radiations in an ATM-dependent pathway [68]. In brain cells, PP1-HDAC1 containing complexes are central actors in the modulation of the epigenetic histone code at the promoters of genes implied in memory formation [69].

Although it is likely that many other phosphorylation sites will be identified in the future, phosphorylation of HDAC1 and HDAC2 has been biochemically well characterized so far, but a whole comprehension of its biological roles is still elusive. It is likely that the scenario will become more and more sophisticated in the future, as long as new PTMs and the crosstalk between them will be discovered.

4.2. Acetylation. Histone deacetylases are the main actors in the acetylation network that takes place in cells, but they are themselves regulated by acetylation.

HDAC1 can be acetylated on six lysines: K218, K220, K432, K438, K439, and K441. It was shown that in cells, a subpopulation of HDAC1 is acetylated by the acetyltransferase p300 at the promoter of glucocorticoidreceptor (GR) driven genes one hour after binding of the hormone. Acetylation of HDAC1 reduces dramatically its enzymatic and repression activities in vitro and in vivo. HDAC1 acts as a coactivator of GR activation of promoters; consequently, a mechanism was proposed that after gene activation induced by the hormone, p300 is recruited at the promoter, and HDAC1 becomes acetylated and inactivated to allow the attenuation of the signal and the restoration of the transcriptional steady-state levels [70].

On the contrary, HDAC2 is not acetylated in vitro by p300 even if five out of the six lysines acetylated on HDAC1 are conserved [71]. The only lysine not conserved is K432 (arginine R433 in HDAC2), which was shown to be the critical residue for HDAC1 acetylation [70]. The conversion of R433 of HDAC2 into lysine rendered HDAC2 acetylated by p300 in vitro although to a lesser extent compared to HDAC1 [71]. Strikingly, the substitution of the C-terminal tail of HDAC2 with the tail of HDAC1 determined a full acetylation of HDAC2, compared to HDAC1 wild type. Vice versa, HDAC1 bearing the C-terminal tail of HDAC2 was totally refractory to acetylation by $\mathrm{p} 300$. Acetylation of HDAC1 not only reduces the activity of HDAC1 itself, but acts in trans also on HDAC2 both in vitro and in vivo on the HDAC1/HDAC2 heterodimers of the Sin3a, NuRD, and CoREST complexes. These observations clearly show how single differences in key residues of HDAC1 and HDAC2 can affect their PTM code. More importantly, they highlight the crucial role of the C-terminal region in the differential regulations of the enzymatic activity of the two enzymes. 
Based on these experimental data, we can speculate that C-terminal domain of HDAC1 and HDAC2, which is not conserved in the other, more divergent class I HDAC3 and HDAC8 [3, 72], has evolved as a molecular platform to specify and finely tune the functions of two highly related proteins in the complex networks of mammalian systems.

Recent work highlights that HDAC2 binds the acetyltransferase CBP only in response to cigarette smoke treatment and is, therefore, acetylated. This acetylation relies on previous CKII-dependent phosphorylation, since the phosphorylation quadruple mutant is less acetylated after CS treatment. This is one of the first instances of a crosstalk between different PTMs acting on HDAC2 (see Figure 2). Opposite to what observed for acetylation of HDAC1 by $\mathrm{p} 300$, acetylation of HDAC2 by CBP increases its transrepressional activity in luciferase-reporter assays [55]. These observations also suggest that the action of different HATs on single HDACs may result in different biological outcomes, creating a multilevel network of regulation.

4.3. Ubiquitination. Ubiquitin (Ub) is a small protein conjugated to lysine residues of target substrates through an isopeptide bond, as a single monomer or as a polyubiquitin chain. The reaction involves a well-defined three-step enzymatic cascade (E1 activating enzymes, E2 conjugating enzymes and E3 ubiquitin ligases). Polyubiquitination mainly targets proteins for degradation via the proteasome machinery, while monoubiquitination acts as a signal for different biological outputs [36].

Both HDAC1 and HDAC2 have been reported to be polyubiquitinated in vitro and in vivo [75-77]. HDAC1 is ubiquitinated and rapidly degraded by a proteasomedependent mechanism in presence of the antiproliferative agent quinidine [78]. Gaughan and colleagues were the first to describe a Ub E3 ligase for HDAC1 in vivo [79]. They showed that HDAC1 is found in complex with the androgen receptor (AR) protein and the $\mathrm{Ub} \mathrm{E} 3$ ligase $\mathrm{Mdm} 2$ at the promoter of AR-driven genes upon hormone treatment in prostate cancer cells. Ubiquitinated forms of HDAC1 were detected only after hormone treatment and were correlated to the Ub E3 ligase activity of $\mathrm{Mdm} 2$. Mdm2 also ubiquitinates AR protein, and HDAC1 cooperates with Mdm2 possibly through deacetylation of critical lysines of $A R$ protein and thus reducing transcription of the downstream gene. HDAC1 ubiquitination by $\mathrm{Mdm} 2$ may represent a further level of fine regulation at the promoter, reducing HDAC1 protein levels and likely exposing the promoter for a second round of active transcription.

HDAC1 ubiquitination and proteasome-dependent degradation was recently shown to have significant biological relevance. Work by $\mathrm{Oh}$ and colleagues identified a functional interaction between HDAC1 and the Chfr Ub ligase. Chfr downregulates HDAC1 levels by directly binding and ubiquitinating HDAC1. This mechanism correlates with increased invasiveness and metastatic potential of prostate and breast cancer cell lines [80]. Selective ubiquitination and depletion of HDAC1, but not of other class I HDACs including HDAC2, is also a critical step of the proinflammation response activated by tumor necrosis factor- $\alpha$ (TNF $\alpha$ ) through the IKK2 signaling pathway [81]. Upon binding of $\mathrm{TNF} \alpha$ to its receptor, the IKK complex (IKK1/IKK2/NEMO) phosphorylates the $\mathrm{IkB} \alpha$ protein, which then releases $\mathrm{NF}-\mathrm{k} \beta$ protein. Free NF-k $\beta$ translocates from the cytoplasm to the nucleus where it acts as a transcription factor activating a plethora of inflammatory, antiapoptotic and proliferative genes. HDAC1 is ubiquitinated after TNF $\alpha$ treatment and degraded via the proteasome machinery, only in the presence of an active IKK2. As a result, a complete loss of HDAC1 occupancy at the promoter of $\mathrm{p} 21^{\mathrm{WAF} / \mathrm{CIP} 1}$ gene is observed. These results provide evidence of a link between inflammatory signaling pathways and modulation of chromatin transcription through PTMs on histone deacetylases.

HDAC2 is specifically ubiquitinated for proteasomal degradation by the Ubc8 E2 conjugase and the RLIM E3 ligase upon treatment with valproic acid, an inhibitor of class I and IIa HDAC enzymatic activities [76].

Interestingly, valproic acid does not affect HDAC1 stability in endometrial stromal sarcoma cells [82]. This work, together with the study by Vashisht Gopal and collaborators [81], suggests the existence of signaling pathways able to discriminate between HDAC1 and HDAC2 at a PTM level to achieve different specific responses in different cellular contexts.

Finally, HDAC2 was shown to be phosphorylated and degraded by the proteasome in bronchial cells upon treatment with cigarette smoke. A possible correlation with the CKII-dependent phosphorylation was proposed [54] (see Figure 2).

4.4. SUMOylation. The conjugation of SUMO proteins (SUMO1, SUMO2, and SUMO3) to substrates mirrors ubiquitin conjugation; an E1-E2-E3 enzymatic cascade adds SUMO proteins to lysines of target substrates. Differently from ubiquitination, the SUMOylation consensus site $\Phi$-K$\mathrm{X}-\mathrm{E}$ (where $\Phi$ is an aliphatic residue, preferably $\mathrm{L}, \mathrm{I}$ or $\mathrm{V}, \mathrm{K}$ is lysine, $\mathrm{X}$ is any residue and $\mathrm{E}$ is glutamate) is frequently, even though not always, found on substrates [83].

HDAC1 is SUMOylated by SUMO1 in vivo and in vitro, and by SUMO2/3 in vitro $[75,84,85]$. Two independent works mapped the SUMOylation sites of HDAC1 on lysines K444 and K476, but its biological role is still under investigation. We did not observed an effect of the double SUMOylation mutant K444R, K476R (2R) on HDAC1 enzymatic activity and transcriptional repression [84], while work from De Pinho's laboratory reported a $60 \%$ reduction of transcriptional repression of the $2 \mathrm{R}$ HDAC1 mutant, even if the binding with the Sin3a protein was unaffected.

Moreover, overexpression of wild type HDAC1, but not of the $2 \mathrm{R}$ mutant, induced accumulation of cells in the G2 phase of the cell cycle, suggesting that the SUMOylation mutant of HDAC1 is impaired in some biological functions [75]. Furthermore, other studies have reported that SUMO modification promotes transcriptional repression of HDAC1/HDAC2 containing complexes, such as Elk1or Mi2/NuRD [86, 87]. 


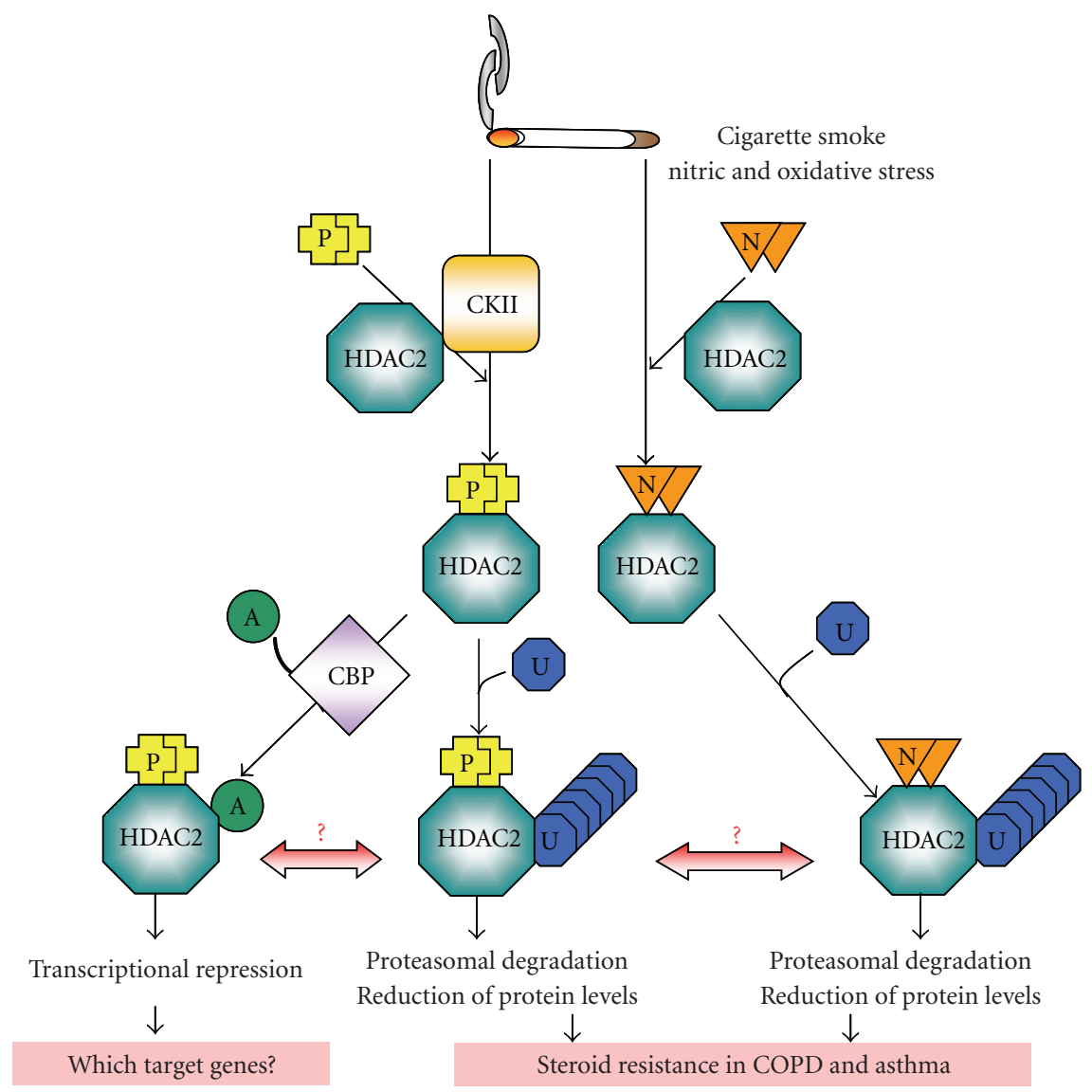

Figure 2: Schematic illustration of the crosstalk between different PTMs on HDAC2 in response to oxidative or nitric stress stimuli. Oxidative or nitric stimuli evoke a complex response in terms of PTMs on HDAC2 that results in a specific biological output [54, 55, 73, 74]. Different PTMs are illustrated by different colors and letters: P (yellow): phosphorylation, A (green): acetylation, U (blue): ubiquitination, and N (orange): nitrosylation. The modification enzymes, when known, are also reported: CKII: casein kinase II, and CBP: CBP histone acetyltransferase. When a mechanistic relation between two different PTMs events is not known, the question mark (?) has been used.

The ability of the $2 \mathrm{R}$ HDAC1 mutant to be polyubiquitinated was also evaluated: although both the wild type and the 2R mutant show consistent ubiquitination, the latter appeared to be ubiquitinated to a lesser extent [75]. If this is due to a mutation of the two lysines, which could serve as target residues for conjugation of ubiquitin, or to a more complex cross regulation between SUMOylation and ubiquitination still remains unknown. It is also worth noticing that SUMOylation of the phospho-null mutant S421A, S423A of HDAC1 was increased respect to the wild-type protein [84]. This finding has not been further investigated, but it may suggest a negative relationship between CKII phosphorylation and SUMOylation.

SUMO proteins are reversibly detached from substrates through the action of SUMO proteases called SENPs (SENP1-7 in mammals) [88].

HDAC1 can be deSUMOylated in vivo by SENP1 [89], a SUMO protease able to cleave both conjugated SUMO1 and SUMO2, but not SUMO3 [90].

HDAC1 represses AR-gene transcription [91] and SENP1 was shown to enhance ligand-dependent transcription of androgen-receptor (AR) driven genes. HDAC1 and HDAC2 were found to complex with SENP1 at the promoter of AR-genes and the transcription-promoting action of SENP1 is carried out through the deSUMOylation of HDAC1. Consistent with this model and with previous results [75], wild type $\mathrm{HDAC} 1$ can repress AR-activity up to $90 \%$ while the $2 \mathrm{R}$ HDAC1 mutant only to $60 \%$ [89].

Up to date, SUMOylation of HDAC2 has not been reported in the literature, but unpublished data in our laboratory have identified lysine 462 as a target site for SUMOylation of HDAC2 by SUMO1 both in vitro and in vivo (C.V. Segre' and S. Chiocca, unpublished data). It is worth noticing that this SUMO consensus site for HDAC2 is not conserved exactly as in HDAC1, but its position in the protein sequence is intermediate between the positions of the two SUMO sites on HDAC1. This might suggest slight differences in the regulation of HDAC1 and HDAC2 by SUMOylation.

Proteins can interact with SUMO also in a noncovalent manner, through a SUMO-Interacting-Motif (SIM) [92]. HDAC2 in particular is recruited by different SUMO-bound transcriptional factors, such as Elk1 and CBP to repress transcription of target genes [86, 93]. SUMOylation of Elk1 
in vitro specifically promotes HDAC2 binding, suggesting the presence of a SIM motif on HDAC2. Finally, recent evidence indicates that HDAC2 promotes SUMOylation of the translation initiation factor eIF4E, modulating the translation of a specific subset of mRNAs [94].

4.5. Nitrosylation. Nitrosylation is a protein modification in which a nitrosyl group (NO) is post-translationally added to an amino acid residue. Nitric oxide (NO) production in cells is catalyzed by enzymes called nitric oxide synthases (NOS), and it represents a key second messenger in a variety of biological processes including neurotransmission, immune defense, apoptosis, and cell motility [95]. NO can rapidly react with the superoxide anion $\mathrm{O}_{2}{ }^{-}$producing the highly oxidant specie peroxynitrite $\left(\mathrm{ONOO}^{-}\right)$, which in turn reacts with amino acids and generates a nitrosylated residue.

Tyrosine nitrosylation was observed in HDAC1, HDAC2, and also HDAC3 in macrophage cells after exposure to cigarette smoke [96]. Another study using human alveolar epithelial cancer cells showed that different tyrosine residues of HDAC2 are nitrosylated after oxidative and nitric stress: Y68, Y73, Y167, Y146, Y173, and Y253. Interestingly, mutation of Y253 abolished the proteasomal-dependent degradation induced by the peroxynitrite generator SIN-1 [73], thus suggesting that tyrosine nitrosylation of HDAC2 is a stress signal to drive specifically its degradation.

In neurons, HDAC2 is s-nitrosylated on cysteines 262 and 274 in response to BDNF-NO signaling. Null mutations of these cysteines did not dramatically affect HDAC2 enzymatic activity, but s-nytrosilation caused dissociation of HDAC2 from the promoters of some neuronal specific genes, such as Fos, Egr1, Vgf, and Nos1 with concomitant increase of histone acetylation and transcriptional activation [97]. Snitrosylation of HDAC2 also plays a role in the pathogenesis of Duchenne muscular dystrophy, where it is correlated with a decrease in HDAC2 enzymatic activity and partial rescue of the myotube differentiation abilities of cells, which are lost in the pathology [98].

Recent work showed that s-nitrosylation of HDAC2 in macrophages following lipopolysaccharide (LPS) stimulation affects the stability of the binding between HDAC2 and MTA1, with consequent dissociation of the NuRD complex from chromatin and activation of transcription of some inflammatory genes like IL1 $\beta$, TNF $\alpha$ and MIP2 [99].

This is another indication that PTMs of HDAC1 and HDAC2 are the first and most important level of regulation, influencing also other levels of regulation such as multiprotein complex formation.

Interestingly, no s-nitrosylation has been reported for HDAC1 or HDAC3 [98], even if the two cysteines are well conserved in all three proteins [48].

These studies suggest an emerging role of s-nytrosilation in regulating the chromatin-associated dynamics of HDAC2, in a very specific way also compared to other class I HDACs, such as the highly related HDAC1.

4.6. Carbonylation. Carbonylation (or alkylation) is a distinctive PTM of redox signaling pathways occurring in cells after oxidative stress. Reactive carbonyl species (RCS) derive from peroxydation of lipids (especially arachidonic acid), which generate $\alpha$ - $\beta$-unsatured aldehydes (e.g., 4 -hydroxy-2nonenal) and $\alpha$ - $\beta$-unsatured ketones (e.g., cyclopentenone prostaglandis). Covalent binding of RCS on cysteinyl thiols of substrate proteins is termed carbonylation [74].

HDAC1, HDAC2, and HDAC3, but not the other class I HDAC8, are carbonylated after treatment with cyclopentenone prostaglandis [100]. The cysteines involved in this PTM have been identified for HDAC1 as Cys 261 and Cys 273, which are conserved in both HDAC2 and HDAC3, but not in HDAC8, which in fact is not modified. Carbonylation of HDAC1 does not impair its intrinsic enzymatic activity, but disrupts its interactions with binding partners and histone substrates in vivo. As a consequence, increased acetylation of lysine 9 of histone $\mathrm{H} 3$ was observed with concomitant induction of transcription of some HDAC1repressed genes, such as HO-1, Gadd45, and HSP70. This paper represents one of the first reports linking redox signaling species such as RCS to modification in chromatin states through a direct modulation of histone deacteylases, resulting in the expression of genes involved in the response of cells to oxidative stresses.

The authors did not formally demonstrate that the corresponding cysteines of HDAC2 and HDAC3 are the carbonylation sites as for HDAC1, but speculated that they are likely to be the target residues of the cyclopentenone prostaglandis covalent binding, based on the evolutionary conservation with HDAC1. It is a reasonable speculation considering that for other PTMs, such as phosphorylation, a similar pattern of modification occurs on corresponding residues of HDAC1 and HDAC2 (see Table 1 and Figure 1). Nevertheless, in the case of redox signaling-associated PTMs, the picture might be more complicated. In fact, HDAC2 is modified by NO on the conserved Cys 262 and Cys 274 [97], but HDAC1 and HDAC3 are not [98], indicating that the simple conservation of the same amino acidic stretch is not always sufficient to have the same PTM. It is likely that other factors, such as cell-type specific interactors, contribute to the final PTM pattern of single HDACs.

\section{Reassembling the Pieces of the Puzzle: The Integration of the Code}

The bulk of work on HDAC1 and HDAC2 PTMs (summarized in Table 1) seems to unveil a complex "code" by which the two proteins can be differentially regulated, despite their high overall similarity and partially redundant roles.

Two "modular" areas of PTMs can be distinguished on HDAC1 and HDAC2: a "tyrosine-cysteine modification" central domain and a "serine-lysine modification" Cterminal domain (see Figures 1(a) and 1(b)).

The vast majority of the modifications reported in the central domain of the two HDACs are nitrosylations and carbonylations in consequence of inflammatory/oxidative stresses; it could be speculated that the central domains of the deacetylases act as a platform for the integration of signals in response to stress stimuli (such as cigarette smoke, LPS, or 
TABLE 1: Summary of the PTM code on HDAC1 and HDAC2. The modification/demodification enzymes, amino acidic sites, and the effects and/or the biological relevance caused by the modification and the citations (refs) are reported.

\begin{tabular}{|c|c|c|c|c|}
\hline \multicolumn{5}{|c|}{ Phosphorylation } \\
\hline & $\begin{array}{c}\text { Kinase/ } \\
\text { Phosphatase }\end{array}$ & Sites & Effects/biological relevance & Refs \\
\hline \multirow{8}{*}{ HDAC1 } & CKII & $\begin{array}{l}\text { S421, S423 in vivo and in } \\
\text { vitro }\end{array}$ & Stimulation of catalytic activity and binding properties & {$[45,48,49]$} \\
\hline & PKA & in vitro & n.r. & {$[45]$} \\
\hline & $\begin{array}{l}\text { PKC, Cdc2 and } \\
\text { MAP kinase }\end{array}$ & $\begin{array}{l}\text { NOT phosphorylated in } \\
\text { vitro }\end{array}$ & l & {$[45]$} \\
\hline & n.r & S406 in vivo & Consequent to viral infection & {$[56,57]$} \\
\hline & n.r. & S393 & identified by mass spectrometry analysis & {$[45,46,51]$} \\
\hline & n.r. & Y221 & identified by mass spectrometry analysis & {$[46]$} \\
\hline & $\begin{array}{c}\text { Protein } \\
\text { phosphatase } 1\end{array}$ & in vitro & n.r. & {$[62]$} \\
\hline & $\lambda$-phosphatase & in vitro & n.r. & {$[62]$} \\
\hline \multirow{9}{*}{ HDAC2 } & \multirow{4}{*}{ CKII } & \multirow{4}{*}{$\begin{array}{l}\text { S394, S422, S424 in vivo } \\
\text { and in vitro }\end{array}$} & Stimulation of catalytic activity and binding properties & {$[50]$} \\
\hline & & & Differential localization on chromatin & {$[47,52]$} \\
\hline & & & Transcriptional regulation of SM $22 \alpha$ in vascular cells & {$[53]$} \\
\hline & & & Transcriptional regulation in bronchial cells & {$[54,55]$} \\
\hline & $\begin{array}{c}\text { PKA, PKC, and } \\
\text { PKG }\end{array}$ & $\begin{array}{l}\text { NOT phosphorylated in } \\
\text { vitro }\end{array}$ & 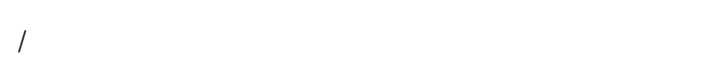 & {$[50]$} \\
\hline & n.r. & S411 in vivo & n.r. & {$[50]$} \\
\hline & n.r. & S407 in vivo & Consequent to viral infection & {$[56-58]$} \\
\hline & $\begin{array}{c}\text { Protein } \\
\text { phosphatase } 1\end{array}$ & in vitro & n.r. & {$[62]$} \\
\hline & $\lambda$-phosphatase & in vitro & n.r. & {$[62]$} \\
\hline \multicolumn{5}{|c|}{ Acetylation } \\
\hline & HAT/HDAC & Sites & Effects/biological relevance & Refs \\
\hline \multirow[t]{2}{*}{ HDAC1 } & p300 (HAT) & $\begin{array}{l}\text { K218, K220, K432, K438, } \\
\text { K439, K441 }\end{array}$ & Transcriptional attenuation of GR-driven genes & {$[70]$} \\
\hline & p300 (HAT) & in vivo and in vitro & Reduction of catalytic activity and binding properties & {$[71]$} \\
\hline \multirow{2}{*}{ HDAC2 } & CBP (HAT) & n.r. & Increase of transcriptional repressive activity & {$[55]$} \\
\hline & p300 (HAT) & NOT acetylated & 1 & {$[71]$} \\
\hline \multicolumn{5}{|c|}{ Ubiquitination } \\
\hline & $\mathrm{E} 1 / \mathrm{E} 2 / \mathrm{E} 3$ & Sites & Effects/biological relevance & Refs \\
\hline \multirow{4}{*}{ HDAC1 } & Mdm2 E3 ligase & n.r. & Modulation of transcription at AR-driven promoters & {$[79]$} \\
\hline & Chfr E3 ligase & n.r. & $\begin{array}{l}\text { Increase of invasiveness and metastatic potential of } \\
\text { cancer cells }\end{array}$ & {$[80]$} \\
\hline & n.r. & n.r. & Response to the antiproliferative agent quinidine & {$[78]$} \\
\hline & n.r. & n.r. & Response to TNF $\alpha$ stimulation via IKK2 signalling & {$[81]$} \\
\hline \multirow{3}{*}{ HDAC2 } & $\begin{array}{l}\text { Ubc8 E2 } \\
\text { conjugase }\end{array}$ & n.r. & Response to valproic acid treatment & {$[76]$} \\
\hline & RLIM E3 ligase & n.r. & & \\
\hline & n.r. & n.r. & Response to oxidative and nitric stress & {$[54,73]$} \\
\hline \multicolumn{5}{|c|}{ SUMOylation } \\
\hline & $\begin{array}{c}\text { SUMO } \\
\text { protein/proteases }\end{array}$ & Sites & Effects/biological relevance & Refs \\
\hline \multirow{3}{*}{ HDAC1 } & SUMO1 & $\begin{array}{l}\text { K444, K476 in vivo and in } \\
\text { vitro }\end{array}$ & Promotion of transcriptional repression [70] & {$[71,81]$} \\
\hline & SUMO $2 / 3$ & in vitro & n.r. & {$[82]$} \\
\hline & $\begin{array}{l}\text { SENP1 SUMO1/2 } \\
\text { protease }\end{array}$ & n.r. & Enhancement of transcription at AR-driven genes & {$[86]$} \\
\hline
\end{tabular}


TABle 1: Continued.

\begin{tabular}{|c|c|c|c|c|}
\hline \multicolumn{5}{|c|}{ Phosphorylation } \\
\hline & $\begin{array}{c}\text { Kinase/ } \\
\text { Phosphatase }\end{array}$ & Sites & Effects/biological relevance & Refs \\
\hline HDAC2 & SUMO1 & K462 in vivo and in vitro & n.r. & $\begin{array}{l}\text { unpublished } \\
\text { data } \\
\text { (C. V. Segre' and } \\
\text { S. Chiocca) }\end{array}$ \\
\hline \multicolumn{5}{|c|}{ Nitrosylation } \\
\hline & Enzyme & Sites & Effects/biological relevance & Refs \\
\hline \multirow{2}{*}{ HDAC1 } & n.r. & Tyrosine(s) & Response to cigarette smoke in macrophages & {$[96]$} \\
\hline & n.r. & NOT s-nitrosylated & 1 & {$[98]$} \\
\hline \multirow{7}{*}{ HDAC2 } & n.r. & Tyrosine(s) & Response to cigarette smoke in macrophages & {$[96]$} \\
\hline & \multirow[t]{2}{*}{ n.r. } & $\begin{array}{l}\text { Y68, Y73, Y167, Y146, } \\
\text { Y173, Y253 }\end{array}$ & $\begin{array}{l}\text { Response to oxidative and nitric stress in bronchial cells } \\
\text { Signal for proteasomal degradation (Y253) }\end{array}$ & {$[73]$} \\
\hline & & $\begin{array}{l}\text { C262, C274 } \\
\text { (s-nitrosylation) }\end{array}$ & $\begin{array}{l}\text { Response to BDNF-NO signalling in neurons } \\
\text { Promotion of dissociation from Fos, Egr1, Vgf, and } \\
\text { Nos1 promoters }\end{array}$ & {$[97]$} \\
\hline & n.r. & s-nitrosylation & $\begin{array}{l}\text { Partial rescue of myotube differentiation in Duchenne's } \\
\text { cells }\end{array}$ & \multirow[t]{2}{*}[98]{} \\
\hline & \multirow{3}{*}{ n.r. } & \multirow{3}{*}{ s-nitrosylation } & Decrease of the enzymatic activity & \\
\hline & & & Dissociation of the NuRD complex & \multirow[t]{2}{*}[99]{} \\
\hline & & & $\begin{array}{l}\text { Activation of } \mathrm{L} 1 \beta \text {, TNF } \alpha \text {, and MIP } 2 \text { genes in } \\
\text { macrophages }\end{array}$ & \\
\hline \multicolumn{5}{|c|}{ Carbonylation } \\
\hline & Enzyme & Sites & Effects/biological relevance & Refs \\
\hline \multirow[t]{2}{*}{ HDAC1 } & \multirow[t]{2}{*}{ n.r. } & \multirow[t]{2}{*}{ C261, C273 } & $\begin{array}{l}\text { Disruption of binding with transcriptional partners } \\
\text { and histone substrates }\end{array}$ & \multirow[t]{2}{*}[100]{} \\
\hline & & & $\begin{array}{l}\text { Transcriptional derepression of the HO- } 1 \text {, Gadd } 45 \text {, and } \\
\text { HSP70 genes }\end{array}$ & \\
\hline HDAC2 & n.r. & carbonylated & n.r. & {$[100]$} \\
\hline
\end{tabular}

S: serine, Y: tyrosine, K: lysine, C: cysteine, n.r.: not reported in literature or not investigated, HAT: histone acetyltransferase, HDAC: histone deacetylase, E1/E2/E3: ubiquitin enzymatic cascade (see text).

RCS-induced inflammation) or to peculiar signaling pathway (such as the BDNF-NO axis). On the other hand, a broader spectrum of PTMs occurs on the C-terminal domains in response to a variety of different signaling pathways (such as hypoxia, hormone stimulation, extracellular signaling). This modular organization highlights the potential of this post-translational code for the modulation of HDAC1 and HDAC2 functions in many cellular pathways or biological responses to different stimuli.

As it was described in this paper, a broad number of single PTMs occur on HDAC1 and HDAC2 but strikingly very few examples of the interplay among them have been reported so far. The only reported example of PTM crosstalk occurs on HDAC2 in human bronchial epithelial cells when injured with cigarette smoke (CS) [55], (see Figure 2). In response to $\mathrm{CS}, \mathrm{HDAC} 2$ associates with $\mathrm{CKII}$, which in turn induces a strong phosphorylation of HDAC2 itself. CS also induces the formation of a complex between HDAC2 and the acetyltransferase CBP with consequent HDAC2 acetylation and increase in its transcriptional repressive activity. An HDAC2 quadruple mutant for all the CKIIdependent sites is far less acetylated than the wild type in response to $\mathrm{CS}$, showing that acetylation of HDAC2 relies on its CKII phosphorylation. Previous work from the same group [54] had also reported CKII dependent degradation of HDAC2 after CS exposure, and a global model was proposed. Cigarette smoke induces a bulk of phosphorylation of HDAC2, which determines its association with repressor partners and enhances its acetylation via $\mathrm{CBP}$, contributing to further increase its repressive activities. On the other hand, CS-induced phosphorylation induces ubiquitination and degradation of $\mathrm{HDAC} 2$; reduction levels of HDAC2 are associated with steroid resistance in COPD and asthma [101], suggesting a mechanistic explanation for this resistance in smoker patients. It will be interesting in the future to verify which genes are repressed in bronchial cells in response to CS-induced phosphoacetylation of HDAC2 and if acetylation is linked to ubiquitination, or if they are two distinct parallel pathways driven by CKII phosphorylation.

Finally, oxidative and nitric stress induce nitrosylation on tyrosine 253 of HDAC2 in bronchial cells, which represents a signal for its proteasomal degradation [73]. Its possible link with CS-induced-CKII-phosphorylation and degradation has not been investigated. Nevertheless, it is clear that 
cigarette smoke (and similar stress stimuli) evokes a complex PTM response on HDAC2, in which both the modular modification domains, the central and the C-terminal ones, are involved and cross interact. It is a step forward in the elucidation of the PTM code, but more investigation is still required to unveil the global panorama.

Another possible interplay between PTMs involves SUMOylation and CKII-phosphorylation of HDAC1. We have observed that the CKII phosphorylation mutant S421A, S423A is more widely SUMOylated than the wild-type HDAC1 [84]. This suggests a negative regulation of SUMOylation by the action of CKII, possibly as a consequence of the lost catalytic activity and binding properties of HDAC1 S421A and S423A, but the molecular mechanisms and the physiological role have not been investigated.

Finally, it was shown by two independent groups that ubiquitinated [79] and SUMOylated [89] forms of HDAC1 can be found at promoters of AR-driven genes and that both these modifications of HDAC1 contribute to the fine modulation of transcription. It will be challenging to investigate whether a crosstalk between SUMOylation and ubiquitination of HDAC1 exists at androgen-responsive promoters and to which extent this interplay affects gene transcription.

\section{Conclusion: Why Deciphering the Code?}

Class I histone deacetylases, and HDAC1 and HDAC2 in particular, have broad expression in many tissues and play a crucial role in cell cycle progression and proliferation [20]. HDAC1 and HDAC2 are deregulated in many cancers and are emerging as the main deacetylases involved in aberrant pathways of tumor cells in humans. Overexpression of HDAC1 is reported for gastric [102, 103], pancreatic [104, 105], colorectal [106], hepatocellular [107], and prostatic [108] carcinomas. HDAC2 plays a major role in APC-colon cancer; its overexpression is present already at the early polyp stage [109]. High levels of HDAC2 are found also in cervical dysplasia and invasive carcinomas [105]. High expression levels of both HDAC1 and HDAC2, together with HDAC3, have been associated with advanced stages of disease in prostate, gastric and colorectal cancers $[105,106]$.

Given the involvement of deacetylases in cancers, inhibitors of HDACs (HDACi) have been looked at as promising anticancer drugs and several classes of HDACi display potent anticancer activities in vitro. HDACi affect tumor cell growth and survival through induction of cell cycle arrest, block of angiogenesis, increase of antigenicity of tumor cells and induction of apoptosis [110]. A large number of clinical trials using different HDACi have shown their promising antitumor response in vivo; two of them have been approved by US FDA for the treatment of T-cell cutaneous lymphoma; suberoylanilide hydroxamic acid (SAHA, brand name vorinostat) in 2006 [111] and romidepsin (brand name istodax) in 2009 [112].

Unfortunately, their use is still restricted to specific types of cancer and adverse side effects have also been recorded, in particular to the blood system [113]. One of the main reasons may be the fact that HDACi are not HDAC-isoform specific and this, given the differentiation of roles of single HDACs in different normal and cancer tissues, may represent a considerable limitation to their use in clinical settings.

HDAC1 and HDAC2 are not only relevant in cancer etiology, but are also important in other human diseases. An abnormal transcriptional repression of the growth factor BDNF by HDAC1/HDAC2 containing complexes is found in cortical neurons expressing the mutant huntingtin protein typical of the neurodegenerative Huntington disease [114]. HDAC2 was recently shown to be a crucial regulator of aberrant genetic programs in renal fibrosis associated with diabetes [115]. Finally, HDAC2 is involved in lung diseases such as COPD (as discussed above) and asthma [116].

It is clear that the integration of all the PTMs in a coherent and global code is a priority for understanding the regulatory networks operating on these key enzymes in different cellular, developmental, physiologic, and pathologic phenomena, with relevant clinical implications for a variety of human diseases.

\section{Acknowledgments}

The authors thank all IFOM-IEO campus facilities and all laboratory members. Work in S. C. laboratory is supported by grants from AIRC (Associazione Italiana per la Ricerca sul Cancro) and from the Italian Ministry of Health.

\section{References}

[1] X.-J. Yang and E. Seto, "HATs and HDACs: from structure, function and regulation to novel strategies for therapy and prevention," Oncogene, vol. 26, no. 37, pp. 5310-5318, 2007.

[2] X.-J. Yang and E. Seto, "The Rpd3/Hdal family of lysine deacetylases: from bacteria and yeast to mice and men," Nature Reviews Molecular Cell Biology, vol. 9, no. 3, pp. 206218, 2008.

[3] I. V. Gregoretti, Y.-M. Lee, and H. V. Goodson, "Molecular evolution of the histone deacetylase family: functional implications of phylogenetic analysis," Journal of Molecular Biology, vol. 338, no. 1, pp. 17-31, 2004.

[4] D. D. Leipe and D. Landsman, "Histone deacetylases, acetoin utilization proteins and acetylpolyamine amidohydrolases are members of an ancient protein superfamily," Nucleic Acids Research, vol. 25, no. 18, pp. 3693-3697, 1997.

[5] A. J. M. De Ruijter, A. H. Van Gennip, H. N. Caron, S. Kemp, and A. B. P. Van Kuilenburg, "Histone deacetylases (HDACs): characterization of the classical HDAC family," Biochemical Journal, vol. 370, no. 3, pp. 737-749, 2003.

[6] M. C. Haigis and L. P. Guarente, "Mammalian sirtuinsemerging roles in physiology, aging, and calorie restriction," Genes and Development, vol. 20, no. 21, pp. 2913-2921, 2006.

[7] L. Gao, M. A. Cueto, F. Asselbergs, and P. Atadja, "Cloning and functional characterization of HDAC11, a novel member of the human histone deacetylase family," Journal of Biological Chemistry, vol. 277, no. 28, pp. 25748-25755, 2002.

[8] A. Villagra, F. Cheng, H.-W. Wang et al., "The histone deacetylase HDAC11 regulates the expression of interleukin 10 and immune tolerance," Nature Immunology, vol. 10, no. 1, pp. 92-100, 2009.

[9] H. Liu, Q. Hu, A. J. D'Ercole, and P. Ye, “Histone deacetylase 11 regulates oligodendrocyte-specific gene expression and 
cell development in OL-1 oligodendroglia cells," GLIA, vol. 57, no. 1, pp. 1-12, 2009.

[10] O. Witt, H. E. Deubzer, T. Milde, and I. Oehme, "HDAC family: what are the cancer relevant targets?" Cancer Letters, vol. 277, no. 1, pp. 8-21, 2009.

[11] C. Hubbert, A. Guardiola, R. Shao et al., "HDAC6 is a microtubule-associated deacetylase," Nature, vol. 417, no. 6887, pp. 455-458, 2002.

[12] J. J. Kovacs, P. J. M. Murphy, S. Gaillard et al., "HDAC6 regulates Hsp90 acetylation and chaperone-dependent activation of glucocorticoid receptor," Molecular Cell, vol. 18, no. 5, pp. 601-607, 2005.

[13] D. Waltregny, W. Glénisson, S. L. Tran et al., "Histone deacetylase HDAC8 associates with smooth muscle $\alpha$-actin and is essential for smooth muscle cell contractility," FASEB Journal, vol. 19, no. 8, pp. 966-968, 2005.

[14] I. Oehme, H. E. Deubzer, D. Wegener et al., "Histone deacetylase 8 in neuroblastoma tumorigenesis," Clinical Cancer Research, vol. 15, no. 1, pp. 91-99, 2009.

[15] S. Bhaskara, B. J. Chyla, J. M. Amann et al., "Deletion of histone deacetylase 3 reveals critical roles in S phase progression and DNA damage control," Molecular Cell, vol. 30, no. 1, pp. 61-72, 2008.

[16] G. Eot-Houllier, G. Fulcrand, Y. Watanabe, L. MagnaghiJaulin, and C. Jaulin, "Histone deacetylase 3 is required for centromeric $\mathrm{H} 3 \mathrm{~K} 4$ deacetylation and sister chromatid cohesion," Genes and Development, vol. 22, no. 19, pp. 2639 2644, 2008.

[17] S. K. Knutson, B. J. Chyla, J. M. Amann, S. Bhaskara, S. S. Huppert, and S. W. Hiebert, "Liver-specific deletion of histone deacetylase 3 disrupts metabolic transcriptional networks," EMBO Journal, vol. 27, no. 7, pp. 1017-1028, 2008.

[18] M. Haberland, R. L. Montgomery, and E. N. Olson, "The many roles of histone deacetylases in development and physiology: implications for disease and therapy," Nature Reviews Genetics, vol. 10, no. 1, pp. 32-42, 2009.

[19] G. Zupkovitz, R. Grausenburger, R. Brunmeir et al., "The cyclin-dependent kinase inhibitor p21 is a crucial target for histone deacetylase 1 as a regulator of cellular proliferation," Molecular and Cellular Biology, vol. 30, no. 5, pp. 1171-1181, 2010.

[20] G. Lagger, D. O’Carroll, M. Rembold et al., "Essential function of histone deacetylase 1 in proliferation control and CDK inhibitor repression," EMBO Journal, vol. 21, no. 11, pp. 2672-2681, 2002.

[21] S. Senese, K. Zaragoza, S. Minardi et al., "Role for histone deacetylase 1 in human tumor cell proliferation," Molecular and Cellular Biology, vol. 27, no. 13, pp. 4784-4795, 2007.

[22] W.-M. Yang, S.-C. Tsai, Y.-D. Wen, G. Fejé, and E. Seto, "Functional domains of histone deacetylase-3," Journal of Biological Chemistry, vol. 277, no. 11, pp. 9447-9454, 2002.

[23] Y. Takami and T. Nakayama, "N-terminal region, C-terminal region, nuclear export signal, and deacetylation activity of histone deacetylase-3 are essential for the viability of the DT40 chicken B cell line," Journal of Biological Chemistry, vol. 275, no. 21, pp. 16191-16201, 2000.

[24] M. J. Kruhlak, M. J. Hendzel, W. Fischle et al., "Regulation of global acetylation in mitosis through loss of histone acetyltransferases and deacetylases from chromatin," Journal of Biological Chemistry, vol. 276, no. 41, pp. 38307-38319, 2001.

[25] L. Alland, G. David, H. Shen-Li et al., "Identification of mammalian Sds3 as an integral component of the
Sin3/histone deacetylase corepressor complex," Molecular and Cellular Biology, vol. 22, no. 8, pp. 2743-2750, 2002.

[26] C. A. Hassig, T. C. Fleischer, A. N. Billin, S. L. Schreiber, and D. E. Ayer, "Histone deacetylase activity is required for full transcriptional repression by $\mathrm{mSin} 3 \mathrm{~A}$, , Cell, vol. 89, no. 3, pp. 341-347, 1997.

[27] Q. Feng and Y. Zhang, "The NuRD complex: linking histone modification to nucleosome remodeling," Current Topics in Microbiology and Immunology, vol. 274, pp. 269-290, 2003.

[28] T. C. Fleischer, U. J. Yun, and D. E. Ayer, "Identification and characterization of three new components of the $\mathrm{mSin} 3 \mathrm{~A}$ corepressor complex," Molecular and Cellular Biology, vol. 23, no. 10, pp. 3456-3467, 2003.

[29] S. A. Denslow and P. A. Wade, "The human Mi-2/NuRD complex and gene regulation," Oncogene, vol. 26, no. 37, pp. 5433-5438, 2007.

[30] A. W. Bruce, I. J. Donaldson, I. C. Wood et al., "Genomewide analysis of repressor element 1 silencing transcription factor/neuron-restrictive silencing factor (REST/NRSF) target genes," Proceedings of the National Academy of Sciences of the United States of America, vol. 101, no. 28, pp. 1045810463, 2004.

[31] A. You, J. K. Tong, C. M. Grozinger, and S. L. Schreiber, "CoREST is an integral component of the CoREST-human histone deacetylase complex," Proceedings of the National Academy of Sciences of the United States of America, vol. 98, no. 4, pp. 1454-1458, 2001.

[32] E. Choi, C. Han, I. Park et al., "A novel germ cell-specific protein, SHIP1, forms a complex with chromatin remodeling activity during spermatogenesis," Journal of Biological Chemistry, vol. 283, no. 50, pp. 35283-35294, 2008.

[33] J. Liang, M. Wan, Y. Zhang et al., "Nanog and Oct4 associate with unique transcriptional repression complexes in embryonic stem cells," Nature Cell Biology, vol. 10, no. 6, pp. 731-739, 2008.

[34] J. Li, J. Wang, Z. Nawaz, J. M. Liu, J. Qin, and J. Wong, "Both corepressor proteins SMRT and N-CoR exist in large protein complexes containing HDAC3," EMBO Journal, vol. 19, no. 16, pp. 4342-4350, 2000.

[35] C. T. Walsh, S. Garneau-Tsodikova, and G. J. Gatto Jr., "Protein posttranslational modifications: the chemistry of proteome diversifications," Angewandte Chemie, vol. 44, no. 45, pp. 7342-7372, 2005.

[36] D. Hoeller, C.-M. Hecker, and I. Dikic, "Ubiquitin and ubiquitin-like proteins in cancer pathogenesis," Nature Reviews Cancer, vol. 6, no. 10, pp. 776-788, 2006.

[37] R. L. Montgomery, C. A. Davis, M. J. Potthoff et al., "Histone deacetylases 1 and 2 redundantly regulate cardiac morphogenesis, growth, and contractility," Genes and Development, vol. 21, no. 14, pp. 1790-1802, 2007.

[38] M. Haberland, A. Johnson, M. H. Mokalled, R. L. Montgomery, and E. N. Olson, "Genetic dissection of histone deacetylase requirement in tumor cells," Proceedings of the National Academy of Sciences of the United States of America, vol. 106, no. 19, pp. 7751-7755, 2009.

[39] R. L. Montgomery, J. Hsieh, A. C. Barbosa, J. A. Richardson, and E. N. Olson, "Histone deacetylases 1 and 2 control the progression of neural precursors to neurons during brain development," Proceedings of the National Academy of Sciences of the United States of America, vol. 106, no. 19, pp. 7876-7881, 2009.

[40] R. H. Wilting, E. Yanover, M. R. Heideman et al., "Overlapping functions of $\mathrm{Hdac1}$ and $\mathrm{Hdac} 2$ in cell cycle regulation 
and haematopoiesis," EMBO Journal, vol. 29, no. 15, pp. 2586-2597, 2010.

[41] B. H. Huang, M. Laban, C. H.-W. Leung et al., "Inhibition of histone deacetylase 2 increases apoptosis and p21Cip1/WAF1 expression, independent of histone deacetylase 1," Cell Death and Differentiation, vol. 12, no. 4, pp. 395-404, 2005.

[42] O. M. Dovey, C. T. Foster, and S. M. Cowley, "Histone deacetylase 1 (HDAC1), but not HDAC2, controls embryonic stem cell differentiation," Proceedings of the National Academy of Sciences of the United States of America, vol. 107, no. 18, pp. 8242-8247, 2010.

[43] J. Taplick, V. Kurtev, K. Kroboth, M. Posch, T. Lechner, and C. Seiser, "Homo-oligomerisation and nuclear localisation of mouse histone deacetylase 1," Journal of Molecular Biology, vol. 308, no. 1, pp. 27-38, 2001.

[44] Y. L. Deribe, T. Pawson, and I. Dikic, "Post-translational modifications in signal integration," Nature Structural and Molecular Biology, vol. 17, no. 6, pp. 666-672, 2010.

[45] R. Cai, P. Kwon, Y. Yan-Neale, L. Sambuccetti, D. Fischer, and D. Cohen, "Mammalian histone deacetylase 1 protein is posttranslationally modified by phosphorylation," Biochemical and Biophysical Research Communications, vol. 283, no. 2, pp. 445-453, 2001.

[46] J. Rush, A. Moritz, K. A. Lee et al., "Immunoaffinity profiling of tyrosine phosphorylation in cancer cells," Nature Biotechnology, vol. 23, no. 1, pp. 94-101, 2005.

[47] J.-M. Sun, Y. C. Hou, and J. R. Davie, "Differential distribution of unmodified and phosphorylated histone deacetylase 2 in chromatin," Journal of Biological Chemistry, vol. 282, no. 45, pp. 33227-33236, 2007.

[48] M. K. H. Pflum, J. K. Tong, W. S. Lane, and S. L. Schreiber, "Histone deacetylase 1 phosphorylation promotes enzymatic activity and complex formation," Journal of Biological Chemistry, vol. 276, no. 50, pp. 47733-47741, 2001.

[49] P. Karwowska-Desaulniers, A. Ketko, N. Kamath, and M. K. H. Pflum, "Histone deacetylase 1 phosphorylation at S421 and S423 is constitutive in vivo, but dispensable in vitro," Biochemical and Biophysical Research Communications, vol. 361, no. 2, pp. 349-355, 2007.

[50] S.-C. Tsai and E. Seto, "Regulation of histone deacetylase 2 by protein kinase CK2," Journal of Biological Chemistry, vol. 277, no. 35, pp. 31826-31833, 2002.

[51] N. Dephoure, C. Zhou, J. Villén et al., "A quantitative atlas of mitotic phosphorylation," Proceedings of the National Academy of Sciences of the United States of America, vol. 105, no. 31, pp. 10762-10767, 2008.

[52] J.-M. Sun, H. Y. Chen, M. Moniwa, D. W. Litchfield, E. Seto, and J. R. Davie, "The transcriptional repressor Sp3 is associated with CK2-phosphorylated histone deacetylase 2," Journal of Biological Chemistry, vol. 277, no. 39, pp. 35783 35786, 2002.

[53] F. Meng, M. Han, B. Zheng et al., "All-trans retinoic acid increases KLF4 acetylation by inducing HDAC2 phosphorylation and its dissociation from KLF4 in vascular smooth muscle cells," Biochemical and Biophysical Research Communications, vol. 387, no. 1, pp. 13-18, 2009.

[54] D. Adenuga, H. Yao, T. H. March, J. Seagrave, and I. Rahman, "Histone deacetylase 2 is phosphorylated, ubiquitinated, and degraded by cigarette smoke," American Journal of Respiratory Cell and Molecular Biology, vol. 40, no. 4, pp. 464473, 2009.

[55] D. Adenuga and I. Rahman, "Protein kinase CK2-mediated phosphorylation of HDAC2 regulates co-repressor formation, deacetylase activity and acetylation of HDAC2 by cigarette smoke and aldehydes," Archives of Biochemistry and Biophysics, vol. 498, no. 1, pp. 62-73, 2010.

[56] A. P. W. Poon, Y. Liang, and B. Roizman, "Herpes simplex virus 1 gene expression is accelerated by inhibitors of histone deacetylases in rabbit skin cells infected with a mutant carrying a cDNA copy of the infected-cell protein no. 0," Journal of Virology, vol. 77, no. 23, pp. 12671-12678, 2003.

[57] M. S. Walters, A. Erazo, P. R. Kinchington, and S. Silverstein, "Histone deacetylases 1 and 2 are phosphorylated at novel sites during Varicella-Zoster virus infection," Journal of Virology, vol. 83, no. 22, pp. 11502-11513, 2009.

[58] M. S. Walters, P. R. Kinchington, B. W. Banfield, and S. Silverstein, "Hyperphosphorylation of histone deacetylase 2 by alphaherpesvirus US3 kinases," Journal of Virology, vol. 84, no. 19, pp. 9666-9676, 2010.

[59] R. B. Pearson and B. E. Kemp, "Protein kinase phosphorylation site sequences and consensus specificity motifs: tabulations," Methods in Enzymology, vol. 200, pp. 62-81, 1991.

[60] S. Pluemsampant, O. S. Safronova, K.-I. Nakahama, and I. Morita, "Protein kinase CK2 is a key activator of histone deacetylase in hypoxia-associated tumors," International Journal of Cancer, vol. 122, no. 2, pp. 333-341, 2008.

[61] Q. Wang, S. Yin, J.-S. Ai et al., "Histone deacetylation is required for orderly meiosis," Cell Cycle, vol. 5, no. 7, pp. 766774, 2006.

[62] S. C. Galasinski, K. A. Resing, J. A. Goodrich, and N. G. Ahn, "Phosphatase inhibition leads to histone deacetylases 1 and 2 phosphorylation and disruption of corepressor interactions," Journal of Biological Chemistry, vol. 277, no. 22, pp. 1961819626, 2002.

[63] G. Canettieri, I. Morantte, E. Guzmán et al., "Attenuation of a phosphorylation-dependent activator by an HDAC-PP1 complex," Nature Structural Biology, vol. 10, no. 3, pp. 175$181,2003$.

[64] Q. Jin, A. Van Eynde, M. Beullens et al., "The protein phosphatase-1 (PP1) regulator, nuclear inhibitor of PP1 (NIPP1), interacts with the polycomb group protein, embryonic ectoderm development (EED), and functions as a transcriptional repressor," Journal of Biological Chemistry, vol. 278, no. 33, pp. 30677-30685, 2003.

[65] M. Yoshida, M. Kijima, M. Akita, and T. Beppu, "Potent and specific inhibition of mammalian histone deacetylase both in vivo and in vitro by trichostatin A," Journal of Biological Chemistry, vol. 265, no. 28, pp. 17174-17179, 1990.

[66] M. H. Brush, A. Guardiola, J. H. Connor, T.-P. Yao, and S. Shenolikar, "Deactylase inhibitors disrupt cellular complexes containing protein phosphatases and deacetylases," Journal of Biological Chemistry, vol. 279, no. 9, pp. 7685-7691, 2004.

[67] C.-S. Chen, S.-C. Weng, P.-H. Tseng, H.-P. Lin, and C.S. Chen, "Histone acetylation-independent effect of histone deacetylase inhibitors on Akt through the reshuffling of protein phosphatase 1 complexes," Journal of Biological Chemistry, vol. 280, no. 46, pp. 38879-38887, 2005.

[68] C. Guo, J. Mi, D. L. Brautigan, and J. M. Larner, "ATM regulates ionizing radiation-induced disruption of HDAC1:PP1:Rb complexes," Cellular Signalling, vol. 19, no. 3, pp. 504-510, 2007.

[69] K. Koshibu, J. Gräff, M. Beullens et al., "Protein phosphatase 1 regulates the histone code for long-term memory," Journal of Neuroscience, vol. 29, no. 41, pp. 13079-13089, 2009.

[70] Y. Qiu, Y. Zhao, M. Becker et al., "HDAC1 acetylation is linked to progressive modulation of steroid receptor-induced 
gene transcription," Molecular Cell, vol. 22, no. 5, pp. 669679, 2006.

[71] Y. Luo, W. Jian, D. Stavreva et al., "Trans-regulation of histone deacetylase activities through acetylation," The Journal of Biological Chemistry, vol. 284, pp. 34901-34910, 2009.

[72] X. -J. Yang and E. Seto, "Collaborative spirit of histone deacetylases in regulating chromatin structure and gene expression," Current Opinion in Genetics and Development, vol. 13, no. 2, pp. 143-153, 2003.

[73] G. O. Osoata, S. Yamamura, M. Ito et al., "Nitration of distinct tyrosine residues causes inactivation of histone deacetylase 2," Biochemical and Biophysical Research Communications, vol. 384, no. 3, pp. 366-371, 2009.

[74] P. A. Grimsrud, H. Xie, T. J. Griffin, and D. A. Bernlohr, "Oxidative stress and covalent modification of protein with bioactive aldehydes," Journal of Biological Chemistry, vol. 283, no. 32, pp. 21837-21841, 2008.

[75] G. David, M. A. Neptune, and R. A. Depinho, "SUMO-1 modification of histone deacetylase 1 (HDAC1) modulates its biological activities," Journal of Biological Chemistry, vol. 277, no. 26, pp. 23658-23663, 2002.

[76] O. H. Krämer, P. Zhu, H. P. Ostendorff et al., "The histone deacetylase inhibitor valproic acid selectively induces proteasomal degradation of HDAC2," EMBO Journal, vol. 22, no. 13, pp. 3411-3420, 2003.

[77] Y. Li, X. Li, and B. Guo, "Chemopreventive agent 3,3'diindolylmethane selectively induces proteasomal degradation of class I histone deacetylases," Cancer Research, vol. 70, no. 2, pp. 646-654, 2010.

[78] Q. Zhou, Z. K. Melkoumian, A. Lucktong, M. Moniwa, J. R. Davie, and J. S. Strobl, "Rapid induction of histone hyperacetylation and cellular differentiation in human breast tumor cell lines following degradation of histone deacetylase1," Journal of Biological Chemistry, vol. 275, no. 45, pp. 35256-35263, 2000.

[79] L. Gaughan, I. R. Logan, D. E. Neal, and C. N. Robson, "Regulation of androgen receptor and histone deacetylase 1 by Mdm2-mediated ubiquitylation," Nucleic Acids Research, vol. 33, no. 1, pp. 13-26, 2005.

[80] Y. M. Oh, Y. E. Kwon, J. M. Kim et al., "Chfr is linked to tumour metastasis through the downregulation of HDAC1," Nature Cell Biology, vol. 11, no. 3, pp. 295-302, 2009.

[81] Y. N. Vashisht Gopal, T. S. Arora, and M. W. Van Dyke, "Tumour necrosis factor- $\alpha$ depletes histone deacetylase 1 protein through IKK2," EMBO Reports, vol. 7, no. 3, pp. 291296, 2006.

[82] A. Hrzenjak, F. Moinfar, M.-L. Kremser et al., "Valproate inhibition of histone deacetylase 2 affects differentiation and decreases proliferation of endometrial stromal sarcoma cells," Molecular Cancer Therapeutics, vol. 5, no. 9, pp. 2203-2210, 2006.

[83] R. Jürgen Dohmen, "SUMO protein modification," Biochimica et Biophysica Acta, vol. 1695, no. 1-3, pp. 113-131, 2004.

[84] R. Colombo, R. Boggio, C. Seiser, G. F. Draetta, and S. Chiocca, "The adenovirus protein Gam1 interferes with sumoylation of histone deacetylase 1," EMBO Reports, vol. 3, no. 11, pp. 1062-1068, 2002.

[85] O. Kirsh, J.-S. Seeler, A. Pichler et al., "The SUMO E3 ligase RanBP2 promotes modification of the HDAC4 deacetylase," EMBO Journal, vol. 21, no. 11, pp. 2682-2691, 2002.

[86] S.-H. Yang and A. D. Sharrocks, "SUMO promotes HDACmediated transcriptional repression," Molecular Cell, vol. 13, no. 4, pp. 611-617, 2004.
[87] Z. Gong, M. Brackertz, and R. Renkawitz, "SUMO modification enhances p66-mediated transcriptional repression of the Mi-2/NuRD complex," Molecular and Cellular Biology, vol. 26, no. 12, pp. 4519-4528, 2006.

[88] E. S. Johnson, "Protein modification by SUMO," Annual Review of Biochemistry, vol. 73, pp. 355-382, 2004.

[89] J. Cheng, D. Wang, Z. Wang, and E. T. H. Yeh, "SENP1 enhances androgen receptor-dependent transcription through desumoylation of histone deacetylase 1," Molecular and Cellular Biology, vol. 24, no. 13, pp. 6021-6028, 2004.

[90] D. Mukhopadhyay and M. Dasso, "Modification in reverse: the SUMO proteases," Trends in Biochemical Sciences, vol. 32, no. 6, pp. 286-295, 2007.

[91] Y. Shang, M. Myers, and M. Brown, "Formation of the androgen receptor transcription complex," Molecular Cell, vol. 9, no. 3, pp. 601-610, 2002.

[92] J. Song, Z. Zhang, W. Hu, and Y. Chen, "Small ubiquitinlike modifier (SUMO) recognition of a SUMO binding motif: a reversal of the bound orientation," Journal of Biological Chemistry, vol. 280, no. 48, pp. 40122-40129, 2005.

[93] H.-Y. Kuo, C.-C. Chang, J.-C. Jeng et al., "SUMO modification negatively modulates the transcriptional activity of CREB-binding protein via the recruitment of Daxx," Proceedings of the National Academy of Sciences of the United States of America, vol. 102, no. 47, pp. 16973-16978, 2005.

[94] X. Xu, J. Vatsyayan, C. Gao, C. J. Bakkenist, and J. Hu, "HDAC2 promotes eIF4E sumoylation and activates mRNA translation gene specifically," Journal of Biological Chemistry, vol. 285, no. 24, pp. 18139-18143, 2010.

[95] B. M. Gaston, J. Carver, A. Doctor, and L. A. Palmer, "Snitrosylation signaling in cell biology," Molecular Interventions, vol. 3, no. 5, pp. 253-263, 2003.

[96] S.-R. Yang, A. S. Chida, M. R. Bauter et al., "Cigarette smoke induces proinflammatory cytokine release by activation of NF- $\kappa \mathrm{B}$ and posttranslational modifications of histone deacetylase in macrophages," American Journal of Physiology, vol. 291, no. 1, pp. L46-L57, 2006.

[97] A. Nott, P. M. Watson, J. D. Robinson, L. Crepaldi, and A. Riccio, "S-nitrosylation of histone deacetylase 2 induces chromatin remodelling in neurons," Nature, vol. 455, no. 7211, pp. 411-415, 2008.

[98] C. Colussi, C. Mozzetta, A. Gurtner et al., "HDAC2 blockade by nitric oxide and histone deacetylase inhibitors reveals a common target in Duchenne muscular dystrophy treatment," Proceedings of the National Academy of Sciences of the United States of America, vol. 105, no. 49, pp. 19183-19187, 2008.

[99] S. B. Pakala, T. M. Bui-Nguyen, S. D.N. Reddy et al., "Regulation of NF- $\kappa$ B circuitry by a component of the nucleosome remodeling and deacetylase complex controls inflammatory response homeostasis," Journal of Biological Chemistry, vol. 285, no. 31, pp. 23590-23597, 2010.

[100] K. Doyle and F. A. Fitzpatrick, "Redox signaling, alkylation (carbonylation) of conserved cysteines inactivates class I histone deacetylases 1,2, and 3 and antagonizes their transcriptional repressor function," Journal of Biological Chemistry, vol. 285, no. 23, pp. 17417-17424, 2010.

[101] K. K. Meja, S. Rajendrasozhan, D. Adenuga et al., "Curcumin restores corticosteroid function in monocytes exposed to oxidants by maintaining HDAC2," American Journal of Respiratory Cell and Molecular Biology, vol. 39, no. 3, pp. 312323, 2008.

[102] J. -H. Choi, H. J. Kwon, B. -I. Yoon et al., "Expression profile of histone deacetylase 1 in gastric cancer tissues," Japanese 
Journal of Cancer Research, vol. 92, no. 12, pp. 1300-1304, 2001.

[103] W. Weichert, A. Röske, V. Gekeler et al., "Association of patterns of class I histone deacetylase expression with patient prognosis in gastric cancer: a retrospective analysis," The Lancet Oncology, vol. 9, no. 2, pp. 139-148, 2008.

[104] K. Miyake, T. Yoshizumi, S. Imura et al., "Expression of hypoxia-inducible factor- $1 \alpha$, histone deacetylase 1 , and metastasis-associated protein 1 in pancreatic carcinoma: correlation with poor prognosis with possible regulation," Pancreas, vol. 36, no. 3, pp. e1-e9, 2008.

[105] W. Weichert, A. Röske, V. Gekeler et al., "Histone deacetylases 1,2 and 3 are highly expressed in prostate cancer and HDAC2 expression is associated with shorter PSA relapse time after radical prostatectomy," British Journal of Cancer, vol. 98, no. 3, pp. 604-610, 2008.

[106] W. Weichert, A. Röske, S. Niesporek et al., "Class I histone deacetylase expression has independent prognostic impact in human colorectal cancer: specific role of class I histone deacetylases in vitro and in vivo," Clinical Cancer Research, vol. 14, no. 6, pp. 1669-1677, 2008.

[107] T. Rikimaru, A. Taketomi, Y.-I. Yamashita et al., "Clinical significance of histone deacetylase 1 expression in patients with hepatocellular carcinoma," Oncology, vol. 72, no. 1-2, pp. 69-74, 2007.

[108] K. Halkidou, L. Gaughan, S. Cook, H. Y. Leung, D. E. Neal, and C. N. Robson, "Upregulation and nuclear recruitment of $\mathrm{HDACl}$ in hormone refractory prostate cancer," Prostate, vol. 59, no. 2, pp. 177-189, 2004.

[109] J. M. Mariadason, "Making sense of HDAC2 mutations in colon cancer," Gastroenterology, vol. 135, no. 5, pp. 14571459, 2008.

[110] P. A. Marks, R. A. Rifkind, V. M. Richon, R. Breslow, T. Miller, and W. K. Kelly, "Histone deacetylases and cancer: causes and therapies," Nature Reviews Cancer, vol. 1, no. 3, pp. 194-202, 2001.

[111] S. Grant, C. Easley, and P. Kirkpatrick, "Vorinostat," Nature Reviews Drug Discovery, vol. 6, no. 1, pp. 21-22, 2007.

[112] "StatBite: FDA oncology drug product approved in 2009," Journal of the National Cancer Institute, vol. 102, p. 219, 2010.

[113] O. A. Botrugno, F. Santoro, and S. Minucci, "Histone deacetylase inhibitors as a new weapon in the arsenal of differentiation therapies of cancer," Cancer Letters, vol. 280, no. 2, pp. 134-144, 2009.

[114] C. Landles and G. P. Bates, "Huntingtin and the molecular pathogenesis of Huntington's disease. Fourth in molecular medicine review series," EMBO Reports, vol. 5, no. 10, pp. 958-963, 2004.

[115] H. Noh, Y. O. Eun, Y. S. Ji et al., "Histone deacetylase-2 is a key regulator of diabetes- and transforming growth factor$\beta 1$-induced renal injury," American Journal of Physiology, vol. 297, no. 3, pp. F729-F738, 2009.

[116] P. J. Barnes, "Histone deacetylase-2 and airway disease," Therapeutic Advances in Respiratory Disease, vol. 3, no. 5, pp. 235-243, 2009. 

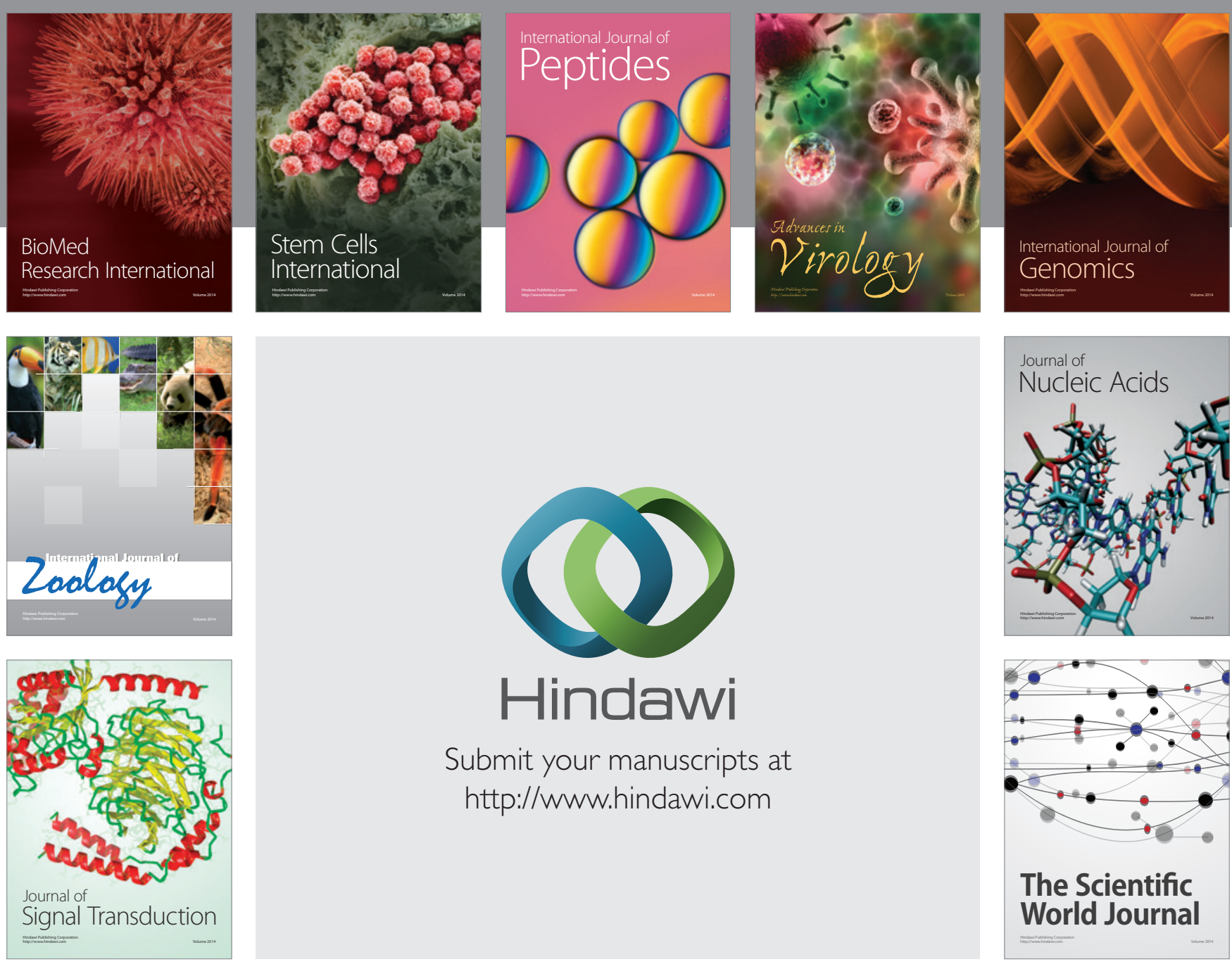

Submit your manuscripts at

http://www.hindawi.com
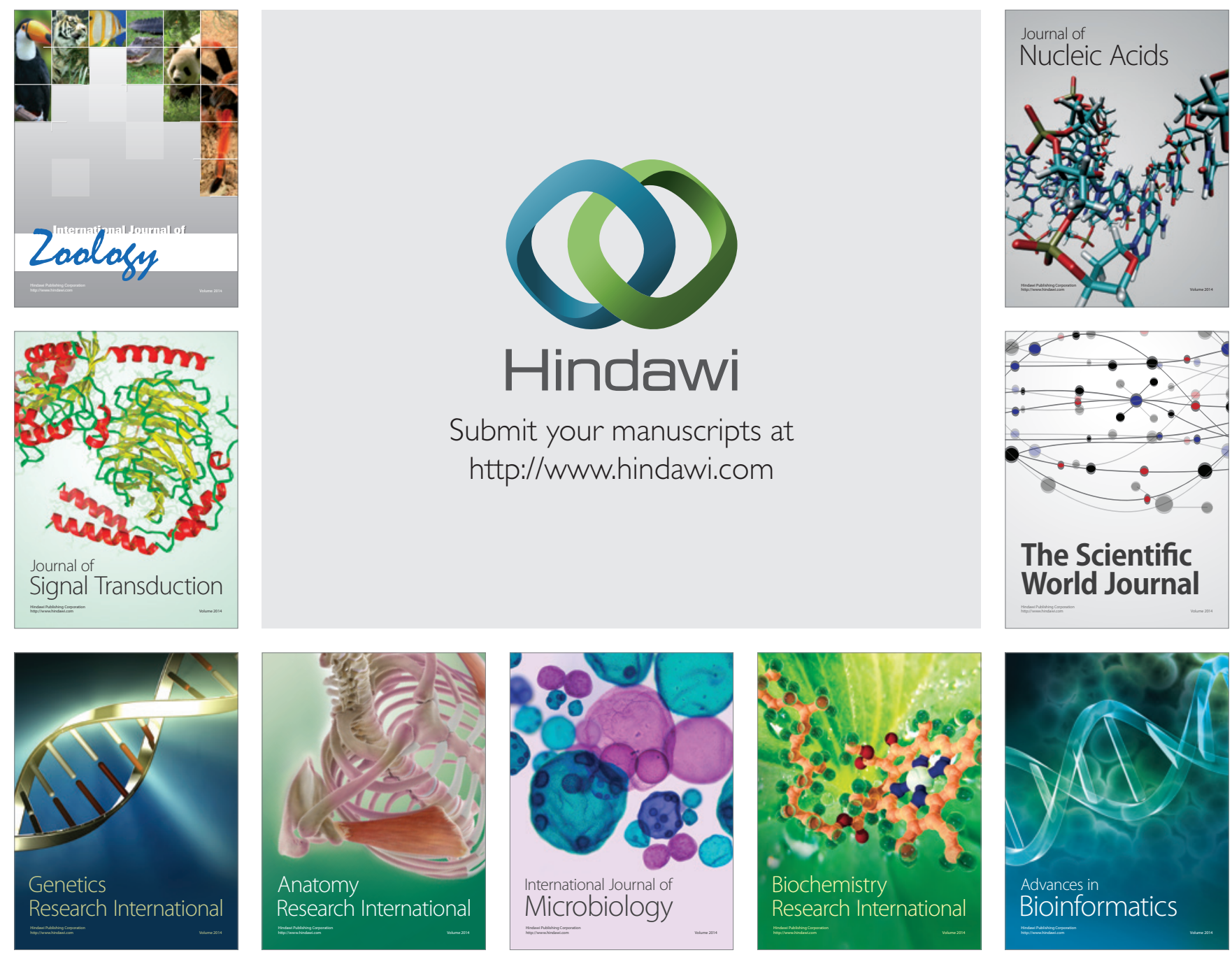

The Scientific World Journal
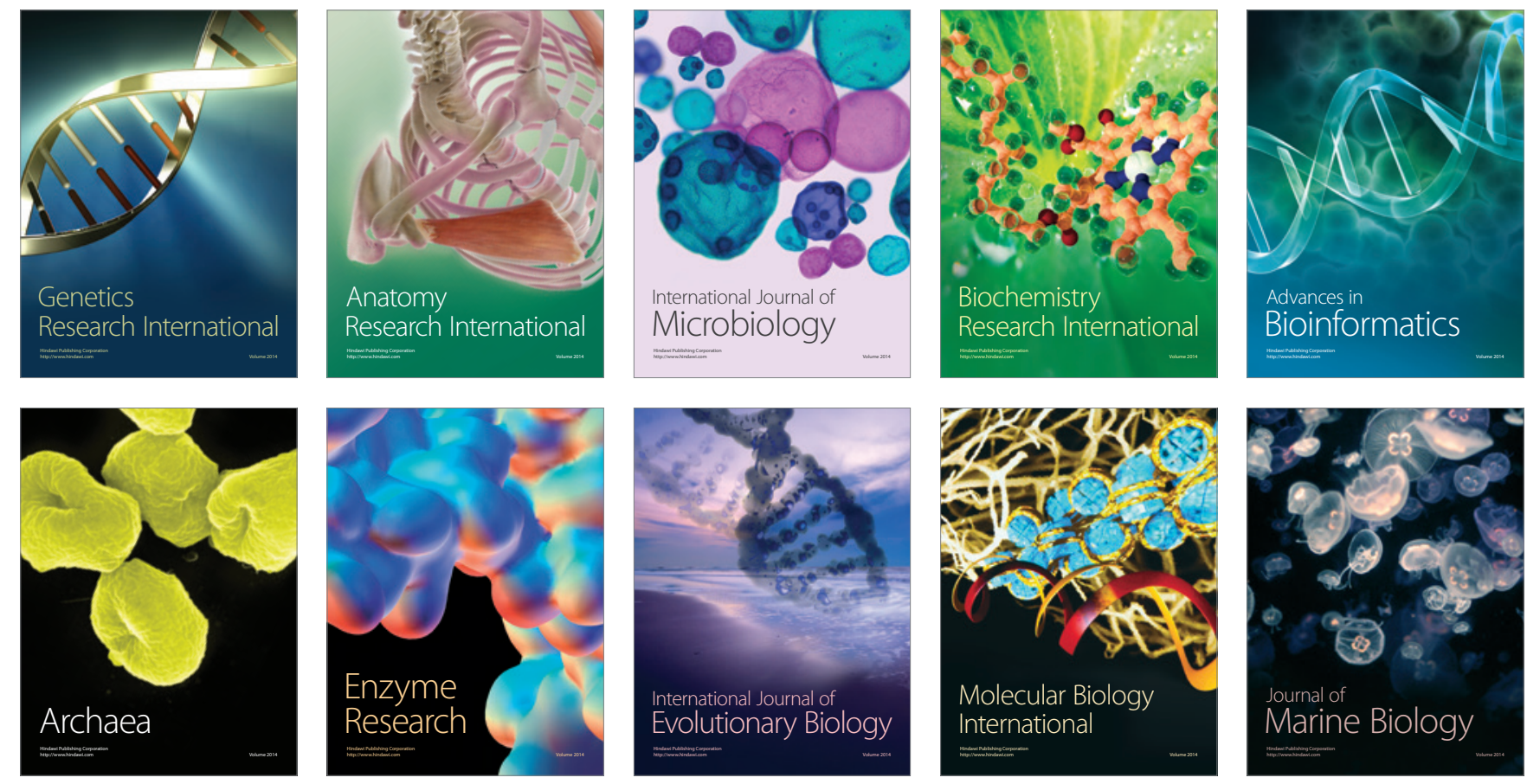